\title{
Transient thermal conduction with variable conductivity using the Meshless Local Petrov-Galerkin method
}

\author{
N.P. Karagiannakis ${ }^{a}$, G.C. Bourantas ${ }^{\text {b,c }}$, A.N. Kalarakis ${ }^{b}$, E.D. Skouras ${ }^{\text {b,a,*, }}$ V.N. Burganos ${ }^{a}$ \\ ${ }^{a}$ Institute of Chemical Engineering Sciences (ICEHT), Foundation for Research and Technology, Hellas (FORTH), GR-26504 Patras, Greece \\ ${ }^{\mathrm{b}}$ TEI of Western Greece, Department of Mechanical Engineering, M. Alexandrou 1, Patras, Greece \\ ${ }^{\mathrm{c}}$ Faculty of Science, Technology and Communication, University of Luxembourg, Luxembourg
}

\section{A R T I C L E I N F O}

\section{Keywords:}

Meshless Petrov-Galerkin

Moving Least Squares

Heat conduction

Spatiotemporally varying conductivity

\begin{abstract}
A B S T R A C T
A numerical solution of the transient heat conduction problem with spatiotemporally variable conductivity in 2D space is obtained using the Meshless Local Petrov-Galerkin (MLPG) method. The approximation of the field variables is performed using Moving Least Squares (MLS) interpolation. The accuracy and the efficiency of the MLPG schemes are investigated through variation of (i) the domain resolution, (ii) the order of the basis functions, (iii) the shape of the integration site around each node, (iv) the conductivity range, and (v) the volumetric heat capacity range. Steady-state boundary conditions of the essential type are assumed. The results are compared with those calculated by a typical Finite Element method. Specific rectangular-type integration sites are introduced during both steady-state and transient MLPG integration, in order to provide complete surface coverage of the domain without overlapping, and the accuracy of the method is demonstrated in all cases studied. Computational efficiency is also investigated with this MLPG method and found to be slower than FE methods during construction stage, but it clearly surpasses that of FEM approaches during the solution stage on a wide parameter range.
\end{abstract}

(c) 2015 Elsevier Inc. All rights reserved.

\section{Introduction}

The subject of heat transfer is of fundamental importance in numerous areas of engineering science involving energy transport in processes driven by temperature differences [1,2]. Transient heat transfer studies provide vital cost-effective and efficient solutions for many modern critical applications, as in modeling of heat transport within biological tissues, which is of direct interest to various therapeutic applications [2]. Variable conductivity can be observed in medicinal applications such as laser ablation of tumors and biological tissues, as well as industrial applications with liquid metals [3], stretching sheets [4], and crystallinity effects on amorphous polymers [5].

The Finite Element method using the Galerkin description has found extensive engineering acceptance as well as vast commercialization, due to its origins in generalized variational principles and its convenience. Compared with its suitability and flexibility in broad use, the Finite Element method has been long criticized for inherent problems such as complex meshing and remeshing problems, connectivity issues, and poor derivatives. Such conventional CFD numerical techniques ordinarily utilized in heat transfer studies still have these inherent deficiencies that lower their effectiveness and reduce

\footnotetext{
* Corresponding author at: Institute of Chemical Engineering Sciences (ICEHT), Foundation for Research and Technology, Hellas (FORTH), GR-26504 Patras, Greece.

E-mail address: Eugene.Skouras@iceht.forth.gr (E.D. Skouras).
} 
their applicability to practical applications, particularly those involving complex transient problems. The main source of deficiency is related to the requirement of a well-organized and connected mesh in the problem domain and the boundaries. The mesh creation can be highly difficult, usually requiring time-consuming user involvement. Domain mesh generation can be avoided with the application of Boundary Element Methods (BEM), another class of methods broadly applied in heat transfer engineering. However, BEM is usually constrained to cases where the infinite space fundamental solution for the differential operator is available, and the evaluation of the unknown field variable and/or its gradients at any single point within the domain of the problem involves tedious calculations of integrals over the global boundaries. Both FEM and BEM unavoidably have to handle the nonlinear terms during the solution of nonlinear cases.

A more attractive alternative for such tasks is a meshless discretization approach using finite point approximations, which are gradually becoming well-established in recent years. Several meshless methods have been reported thus far, such as the Diffuse Element Method (DEM) [6], the Element Free Galerkin (EFG) method [7-9], and the Reproducing Kernel Particle (RKP) method [10]. Meshless methods have undergone extensive development recently, largely due to their inherent potential of handling engineering problems with complex and irregular geometries more efficiently than standard computational techniques [11]. Meshless numerical techniques rely on local type interpolation on either regularly or irregularly distributed spatial points, usually called nodes. Their main advantage over traditional mesh-based numerical methods is the fact that usually there is no need for a mesh and for definition of the connectivity between adjacent elements. In this way, the effort devoted to mesh generation is reduced considerably, since PDE linearization and interpolation is performed in terms of the nodal points distributed on the problem domain. However, most of these methods still need "shadow" elements to integrate over the entire domain of the problem for integral properties, such as energy or fluxes, and so far, have been applied mainly to solve linear problems.

Linear heat transfer problems have been addressed with meshless methods in a number of heat conduction studies. The transient heat conduction behavior of functionally graded anisotropic materials with continuously variable material properties have been investigated with the application of meshless local boundary integral equations with Moving Least-Squares approximations using Laplace transformation for the time integration of 2D problems [12,13], whereas anisotropic linearlygraded transient three-dimensional problems, either axisymmetric [14], or fully 3D [15], have been efficiently tackled in a similar fashion using Meshless Local Petrov-Galerkin methods.

This truly meshless formulation based on the recently developed [16] local symmetric weak form with the Local PetrovGalerkin approach is proposed here to solve transient non-linear heat conduction problems. The essential boundary conditions in the present formulation were imposed by 1st order MLS description. This method does not need elements or meshes, either for interpolation purposes, or for integration purposes. All integrals are carried out only on circles (in 2-D, or spheres in 3-D) centered at each point in question, or in rectangles (in 2-D, or orthorhombic parallelepipeds in 3-D). The present method is also more flexible and easier in dealing with nonlinear problems than the conventional FEM, EFG and BEM.

In the present work, 2-D thermal conduction problems with spatiotemporally variable conductivity are solved (Fig. 1(a)), although the methodology can be straightforwardly extended to 3-D applications. The Meshless Local Petrov-Galerkin (MLPG) method is used to discretize the problems using Moving Least-Squares (MLS) approximations. The effect of several key parameters on the accuracy and efficiency of the numerical solutions is examined, such as (i) the domain resolution (100 px, 200 px, or refinement), (ii) the order of the basis function used (1st or 2nd), (iii) the shape of the integration site around

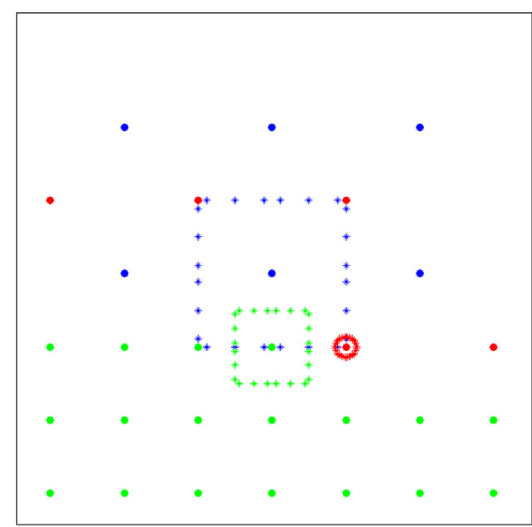

(a)

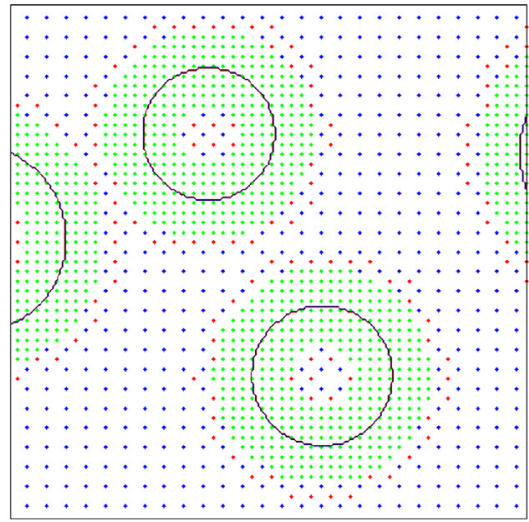

(b)

Fig. 1. (a) Rectangular MLPG integration scheme with the Gauss points of integration around characteristic nodes. (b) Refinement in MLPG scheme. Blue points: basic nodes (at 100 px) with rectangular integration applied; green points: "bulky" refined nodes (at 200 px) near conductivity interfaces with rectangular integration applied; red points: locally refined nodes (at $200 \mathrm{px}$ ) at bulky refinement edges with circular integration applied; stars: limits (Gauss points) of the integration around respective nodes. (For interpretation of the references to color in this figure legend, the reader is referred to the web version of this article.) 
each node used (circular or rectangular), and (iv) the conductivity and (v) the volumetric heat capacity ranges of the media. The collocation-based meshfree approach does not require any grid mesh, except for a set of distributed nodes in the spatial domain [17,18]. Thus, when it comes to complex geometries, this method may have advantages over the conventional FDM and FEM ones, or even more sophisticated methods such as the Lattice-Boltzmann method [18]. The results obtained herein are compared with those of ordinary FEM solutions applied to the same physical problem [19-21].

\section{Methods}

A truly meshless method, based on the MLPG approach and the Moving Least Squares approximation is presented herein, for solving transient heat convection problems with increased accuracy. The present method makes no use of a "finite element mesh", either for purposes of interpolation of the solution variables, or for integration during calculation of energy or fluxes. All integrals can be easily evaluated over regularly shaped domains (in general, spheres in three-dimensional problems) and their boundaries. No post-smoothing technique is required for computing the derivatives of the unknown variable, since the original solution, using the Moving Least Squares approximation, is already sufficiently smooth. Several numerical examples are presented in the paper. In essence, the present meshless method based on the MLPG is found to be a simple, efficient, and attractive method with a great potential in engineering applications.

The solution approximation, $T^{h}(\boldsymbol{x})$, is described following the Moving Least Squares technique [20,22], as the product of a vector of polynomial base, $\boldsymbol{p}(\boldsymbol{x})$, and a vector of coefficients, $\boldsymbol{a}(\boldsymbol{x})$.

$$
T^{h}(\boldsymbol{x})=\boldsymbol{p}^{T}(\boldsymbol{x}) \boldsymbol{a}(\boldsymbol{x})
$$

where $\boldsymbol{p}(\boldsymbol{x}), \boldsymbol{a}(\boldsymbol{x}) \in R^{q}$, and $q$ is the number of monomials in the polynomial bases (here, 3 or 6 ). The local characteristics of the MLS approximation can be considered as a generalization of the traditional least-squares approximation, where the coefficients $\boldsymbol{a}$ are independent of the local position, $\boldsymbol{x}$.

Eq. (1) is referred to as the global least-squares approximation, however, a unique local approximation associated with each point in the domain can also be described. In order to determine the form of $\boldsymbol{a}(\boldsymbol{x})$, a weighted discrete error norm is constructed and minimized.

$$
J(\boldsymbol{x})=\sum_{I=1}^{n} w_{I}(\boldsymbol{x})\left[\sum_{j=1}^{m} p_{j}^{T}\left(\boldsymbol{x}_{I}\right) \boldsymbol{a}(\boldsymbol{x})-T_{I}\right]^{2},
$$

$w_{I}(\boldsymbol{x})$ denotes the weight function, $w_{I}(\boldsymbol{x}) \equiv w_{I}\left(\boldsymbol{x}-\boldsymbol{x}_{I}\right)$, associated with node $I$, while the quantity in brackets is the difference between the local approximation at node $I$ and the field data at node $I, T_{I}$, and $n$ is the number of nodes in the support of $w_{I}(\boldsymbol{x})$. Minimization of Eq. (2) with respect to $\boldsymbol{a}(\boldsymbol{x})$ determines the latter and results in the following linear system

$$
\boldsymbol{A}(\boldsymbol{x}) \boldsymbol{a}(\boldsymbol{x})=\boldsymbol{B}(\boldsymbol{x}) T_{s} .
$$

$T_{S}$ is a vector containing the nodal data, and $A, B \in R^{m \times n}$ are matrix functions of $w_{I}(\boldsymbol{x})$ and $p\left(\boldsymbol{x}_{I}\right)$. Substitution of the solution of Eq. (3) into the global approximation, Eq. (1), completes the least-squares approximation. Derivatives of the shape function, $\phi(\boldsymbol{x})=\mathbf{p}^{T}(\boldsymbol{x}) \mathbf{A}^{-1}(\boldsymbol{x}) \mathbf{B}(\boldsymbol{x})$, can be calculated with the product rule.

The weighted integral of the transient heat conduction equation at the domain $\Omega_{x}$ around point $x$ is given as

$$
\int_{\Omega_{x}}\left[\tilde{\rho} \tilde{c}_{p} \frac{\partial \tilde{T}}{\partial \tilde{t}}-\tilde{\nabla} \cdot(\tilde{k} \tilde{\nabla} \tilde{T})\right] w d \Omega_{x}=0,
$$

with weight function $w=1$ where $x \in \Omega_{\mathrm{x}}$, and 0 elsewhere. Tildes in notation imply dimensional quantities. The 2 nd term (conduction) of Eq. (4) can be integrated by parts and the Gauss theorem can be used to obtain the following weak symmetric formulation:

$$
\int_{\Omega_{x}} \tilde{\nabla} \cdot(\tilde{k} \tilde{\nabla} \tilde{T}) w d \Omega_{x}=\int_{\partial \Omega_{x}} \tilde{k} \tilde{\nabla} \tilde{T}_{\hat{\mathbf{n}}} w d\left(\partial \Omega_{x}\right)-\int_{\Omega_{x}} \tilde{k} \tilde{\nabla} \tilde{T} \tilde{\nabla} w d \Omega_{x},
$$

where $\partial \boldsymbol{\Omega}_{\chi}$ is the boundary of the domain $\boldsymbol{\Omega}_{\chi}$. With the given weight function the last term in Eq. (5) is inherently zero. Time derivatives (1st term of Eq. (4)) can be written in a Crank-Nicholson scheme, however these integrals cannot be downsized using the Gauss theorem. The robustness of the method is increased significantly when the resulting non-linear terms are described in a semi-explicit formulation (at time step $m$ ) of the following type:

$$
\tilde{T}^{(m)} \tilde{\nabla} \tilde{T}^{(m)}=\tilde{T}^{(m)} \tilde{\nabla} \tilde{T}^{(m-1)}+\tilde{T}^{(m-1)} \tilde{\nabla} \tilde{T}^{(m)}-\tilde{T}^{(m-1)} \tilde{\nabla} \tilde{T}^{(m-1)} .
$$

The conductivity, $\tilde{k}$, which is assumed dependent on temperature (first-order dependence in this work, however straightforwardly applicable to more complex descriptions), and the volumetric heat capacity, $\tilde{\rho} \tilde{c}_{p}$, are defined as:

$$
\tilde{k}=\left\{\begin{array}{l}
\tilde{k}_{1}+\tilde{b}_{1} \tilde{T}, x \in \Omega_{1} \\
\tilde{k}_{2}+\tilde{b}_{2} \tilde{T}, x \in \Omega_{2}
\end{array}\right\}
$$




$$
\tilde{\rho} \tilde{c}_{p}=\left\{\begin{array}{l}
\tilde{\rho}_{1} \tilde{c}_{p 1}, x \in \Omega_{1} \\
\tilde{\rho}_{2} \tilde{c}_{p 2}, x \in \Omega_{2}
\end{array}\right\} .
$$

Reduced quantities are determined based on the domain geometry and the boundary conditions,

$$
x=\frac{\tilde{x}}{\tilde{L}}, \quad y=\frac{\tilde{y}}{\tilde{L}}, \quad T=\frac{\tilde{T}-\tilde{T}_{2}}{\tilde{T}_{1}-\tilde{T}_{2}},
$$

and on the physical values of medium 1 , as well:

$$
\begin{aligned}
& t=\frac{\tilde{t} \tilde{k}_{1}}{\tilde{\rho}_{1} \tilde{c}_{1} \tilde{L}^{2}}, \\
& k=\frac{\tilde{k}}{\tilde{k}_{1}}=\left\{\begin{array}{c}
1+b_{1} T, x \in \Omega_{1} \\
k_{r}+b_{2} T, x \in \Omega_{2}
\end{array}\right\}, \\
& \rho c_{p}=\frac{\tilde{\rho} \tilde{c}_{p}}{\tilde{\rho}_{1} \tilde{c}_{p 1}}=\left\{\begin{array}{c}
1, x \in \Omega_{1} \\
\rho c_{p, r}, x \in \Omega_{2}
\end{array}\right\} .
\end{aligned}
$$

Dimensionless characteristic constants of the process are defined as:

$$
k_{r}=\frac{\tilde{k}_{2}}{\tilde{k}_{1}}, \quad b_{1}=\frac{\tilde{b}_{1}}{\tilde{k}_{1}}, \quad b_{2}=\frac{\tilde{b}_{2}}{\tilde{k}_{1}}, \quad b_{r}=\frac{b_{2}}{b_{1}}, \quad \rho c_{p, r}=\frac{\rho_{2} c_{p 2}}{\rho_{1} c_{p 1}}, \quad a_{r}=\frac{k_{r}}{\rho c_{p, r}} .
$$

$k_{r}$ is the ratio of the temperature-independent parts of the conductivity descriptions, while $b_{r}$ is the ratio of the temperaturedependent respective parts. $\Omega_{1}$ and $\Omega_{2}$ are the domains of medium 1 (exterior) and 2 (interior) of the enclosure. If $\Phi$ is the step function defined at the domain of the interior medium (unit there, zero elsewhere), the reduced coefficients can be linearized as:

$$
\begin{aligned}
& k=\left(k_{r}-1\right) \Phi+1+b_{1}\left[\tilde{T}_{2}+\left(\tilde{T}_{1}-\tilde{T}_{2}\right) T\right]\left(b_{r}-1\right) \Phi+b_{1}\left[\tilde{T}_{2}+\left(\tilde{T}_{1}-\tilde{T}_{2}\right) T\right], \\
& \rho c_{p}=\left(\rho c_{p, r}-1\right) \Phi+1 .
\end{aligned}
$$

The iterative form of Eq. (5) can now be written as follows.

$$
\begin{aligned}
& \left(\rho c_{p, r}-1\right) \int_{\Omega_{x}} \Phi \frac{T^{(m)}-T^{(m-1)}}{\Delta t} d \Omega_{X}+\int_{\Omega_{x}} \frac{T^{(m)}-T^{(m-1)}}{\Delta t} d \Omega_{X}-\left[k_{r}-1+b_{1} \tilde{T}_{2}\left(b_{r}-1\right)\right] \int_{\partial \Omega_{x}} \Phi \nabla T_{\mathbf{n}}^{(m)} d\left(\partial \Omega_{x}\right) \\
& \quad-\left(1+b_{1} \tilde{T}_{2}\right) \int_{\partial \Omega_{x}} \nabla T_{\mathbf{n}}^{(m)} d\left(\partial \Omega_{x}\right)-b_{1}\left(\tilde{T}_{1}-\tilde{T}_{2}\right)\left(b_{r}-1\right) \int_{\partial \Omega_{x}} \Phi T^{(m)} \nabla T_{\mathbf{n}}^{(m)} d\left(\partial \Omega_{x}\right)-b_{1}\left(\tilde{T}_{1}-\tilde{T}_{2}\right) \int_{\partial \Omega_{x}} T^{(m)} \nabla T_{\hat{\mathbf{n}}}^{(m)} d\left(\partial \Omega_{x}\right)=0 .
\end{aligned}
$$

After some algebraic manipulation, the final (dimensionless) iterative form of Eq. (5) can be written with all the $m$ th time step terms gathered on the left-hand side, while all other terms, either constant or relative to the previous time step, collected on the other side:

$$
\begin{aligned}
& \left(\rho c_{p, r}-1\right) \int_{\Omega_{x}} \Phi T^{(m)} d \Omega_{x}+\int_{\Omega_{x}} T^{(m)} d \Omega_{x}-\Delta t\left[k_{r}-1+b_{1} \tilde{T}_{2}\left(b_{r}-1\right)\right] \int_{\partial \Omega_{x}} \Phi \nabla T_{\mathbf{n}}^{(m)} d\left(\partial \Omega_{x}\right) \\
& \quad-\Delta t\left(1+b_{1} \tilde{T}_{2}\right) \int_{\partial \Omega_{x}} \nabla T_{\mathbf{n}}^{(m)} d\left(\partial \Omega_{x}\right)-\Delta t b_{1}\left(\tilde{T}_{1}-\tilde{T}_{2}\right)\left(b_{r}-1\right)\left[\int_{\partial \Omega_{x}} \Phi T^{(m-1)} \nabla T_{\mathbf{n}}^{(m)} d\left(\partial \Omega_{x}\right)+\int_{\partial \Omega_{x}} \Phi T^{(m)} \nabla T_{\mathbf{n}}^{(m-1)} d\left(\partial \Omega_{x}\right)\right] \\
& \quad-\Delta t b_{1}\left(\tilde{T}_{1}-\tilde{T}_{2}\right)\left[\int_{\partial \Omega_{x}} T^{(m-1)} \nabla T_{\mathbf{n}}^{(m)} d\left(\partial \Omega_{x}\right)+\int_{\partial \Omega_{x}} T^{(m)} \nabla T_{\mathbf{n}}^{(m-1)} d\left(\partial \Omega_{x}\right)\right]=\left(\rho c_{p, r}-1\right) \int_{\Omega_{x}} \Phi T^{(m-1)} d \Omega_{x} \\
& \quad+\int_{\Omega_{x}} T^{(m-1)} d \Omega_{x}-\Delta t b_{1}\left(\tilde{T}_{1}-\tilde{T}_{2}\right) \int_{\partial \Omega_{x}} T^{(m-1)} \nabla T_{\mathbf{n}}^{(m-1)} d\left(\partial \Omega_{x}\right)-\Delta t b_{1}\left(\tilde{T}_{1}-\tilde{T}_{2}\right)\left(b_{r}-1\right) \int_{\partial \Omega_{x}} \Phi T^{(m-1)} \nabla T_{\mathbf{n}}^{(m-1)} d\left(\partial \Omega_{x}\right) .
\end{aligned}
$$

This linearized set of the form $\mathbf{A}\left(\boldsymbol{T}^{(m-1)}\right) \mathbf{T}^{(m)}=\mathbf{B}\left(\boldsymbol{T}^{(m-1)}\right)$ is used to solve the evolution of the temperature profile iteratively.

The form of the MLPG integration domain is critical to the success of the method. Equidistant, i.e. circular domains around each node are applied in typical MLPG implementations [16], with a radius at specific fraction of the local inter-node distance (usually around 60\%). This approach, though straightforwardly applicable, either does not cover all the area of the domain or it is leading to significant overlapping in the domain integration. This fact can lead to significant stability errors, which accumulate in transient methods. In the present approach, specific rectangular-type areas are used around each node during MLPG integration of Eq. (17), for the first time in MLPG methods (to the authors' knowledge). These rectangular integrations are implemented specifically to lead to zero overlapping during complete coverage of the domain, Fig. 1(a), with notable increase of the stability and robustness of the method, especially at elevated $k_{r}$ ratios. During refinement, 
overlapping cannot be avoided around all the new nodes added without sacrificing significant computational burden. Thus, rectangular integration is used in all nodes but the edges of the refinement, where typical circular integration is used, Fig. 1(b). This effect is confined in the vicinity of the interface layers and, thus, global robustness is kept at elevated levels.

The rectangular integration size is set at the local distance between nodal points $(d)$, in order to be able to cover the entire computational domain without overlapping, while the radius of circular sites is usually set at $0.6 d$, with both rectangular and circular integration types using Gaussian quadratures. A value of $0.1 d$ was used for the nodes at the edges of refinement, much less than the typical value, yet sufficient to minimize overlapping effects. Further details on the process and on the BCs description can be found in [22-24]. The essential boundary conditions are imposed explicit by first order MLS interpolation (details in [23]). Constant temperature $\left(T_{\text {in }}\right.$ and $\left.T_{\text {out }}\right)$ are assumed at two opposite walls of the enclosure (left and right boundaries of Fig. 2(a)), adiabatic conditions are employed at the other two. Two realizations of a square medium filled with two media of different conductivities are investigated, Figs. 2(a) and 3(a), with the same (0.3) surface fraction (volume fraction in 3-D) of each medium across realizations.

The FE methods used here for reference and validation are ordinary weak formulations using Galerkin approaches, with unstructured (trigonal) mesh elements equidistantly distributed over the two media of the enclosure, and boundary layers (local refinement) developed around both sides of the conductivity interfaces.

Regarding the time integration, an adaptive time stepping algorithm was applied to adjust the time step at each iteration, in order to optimize calculations while keeping the error within predefined limits. Using an initial time step of $10^{-6} \mathrm{~s}$, should the $\mathrm{L}_{2}$ norm of the temperature differences became larger than a predefined maximum value (5), the time step was reduced by half and the calculation of the current time value was repeated; otherwise, in case the norm was found smaller than a minimum value (1), the time step for the next iteration was doubled.

\section{Results and discussion}

The evolution of the contours of the reduced temperature profiles of the 1 st realization of the enclosure at early time steps (relative to the time the process reaches steady state, i.e. when the $\mathrm{L}_{2}$ norm of the temperature changes no more than 1\%), are shown in Fig. 2(b) and (d), for two extreme configurations of the relative conductivities of the interior and exterior media of the inlets and matrix, with the corresponding steady state conditions shown in Fig. 2(c) and (e). An ordinary FE method is used as reference for validation, with the maximum resolution of the MLPG method applied, i.e. about 200 px per dimension of the working sample. Similar conditions can be observed in contours of steady-state conditions at the 2nd realization of the sample, Fig. 3(b) and (c).

The error estimations in the temperature profiles at steady state obtained by each method, compared with the ones obtained with the ordinary FE method (the $L_{2}$ norm), locally refined and converged up to $10^{-6}$ precision global accuracy, are shown in Fig. 4(a) and (b), as functions of the conductivity dependence on temperature, $b_{\mathrm{r}}$. The $L_{2}$ norms and the error in the effective reduced conductivity, $k_{\text {eff }}$ error $\equiv \mid k_{\text {eff }}-k_{\text {eff }}\left(\right.$ FEM) $\mid / k_{\text {eff }}($ FEM), for 1 st and 2nd order MLPG methods with local refinement, using rectangular and circular integration sites, are shown in Fig. 5(a) and (b), as functions of the conductivity coefficient, $k_{r}$, which is independent of temperature $\left(b_{r}=0\right)$.

Three discretization levels are displayed; one with 200 nodes along each dimension, one with 100 nodes along each dimension, and a refinement of the previous one, at double the local resolution across the conductivity interfaces. A resolution of 200 nodes is found to be sufficient for the convergence of the method to $10^{-6}$ precision global accuracy, as seen in Fig. 4(a) and (b) where the errors in the $L_{2}$ norms at point $b_{r}=0.1$, divided by the number of points of this MLPG, are in this order of magnitude, while 100 nodes are one (Fig. 4(a)) or two (Fig. 4(b)) orders higher. Local refinement clearly optimizes the solution and offers better description of the effective conductance and the temperature, Figs. 4(a) and (b), 5(a) and (b). The use of 2nd order interpolation increases the computational time without essential improvement on the estimated errors for the transient problems, Fig. 5(a) and (b).

The effect of refinement on the error of the $L_{2}$ norm, for a range of parameters examined at early stages of transient heat conduction simulations are shown in Fig. 6 . These parameters include the temperature-independent conduction range $\left(k_{r}\right)$, the volumetric heat capacity range $\left(\rho c_{p, r}\right)$, and the temperature dependence of conductivity $\left(b_{r}\right)$. Again, local refinement clearly outperforms unrefined simulations as much as an order of magnitude in a wide range of parameters examined in this work.

Temperature-dependent thermal diffusivity coefficients (Eq. (13)) were also extracted from simulations and their effects on the MLPG performance are shown in Fig. 7(a)-(e), as the error in the $L_{2}$ norm compared to similar FEM approach, for gradually increasing volumetric heat capacity. In all cases studied, the refinement procedure use rectangular integration on the majority of nodes and circular integration on the edges of the refinement.

The computational efficiency of the current MLPG implementation was investigated using wall-clock time measurements and it is portrayed here tabulated. Table 1 displays average time estimations of the construction stages, i.e. the calculation of shape functions and integrals, for steady-state and transient MLPG methods, and for mesh generation at the FE methods used in the present work, as well. Increased computational time can be observed for the calculation of surface integrals necessary for transient MLPG calculations, whereas the line integrals, which are used in both steady-state and transient calculations, require significantly less computational time, as expected. However, the shape functions and the line and surface integrals are estimated only once, yet they can be used in many subsequent calculations of the solutions for a wide range of 


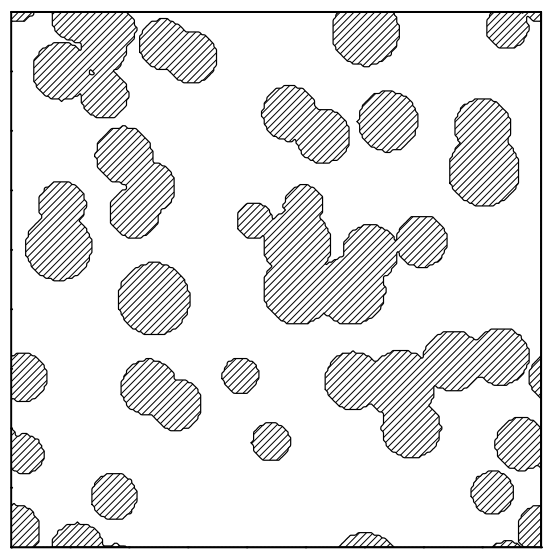

(a)
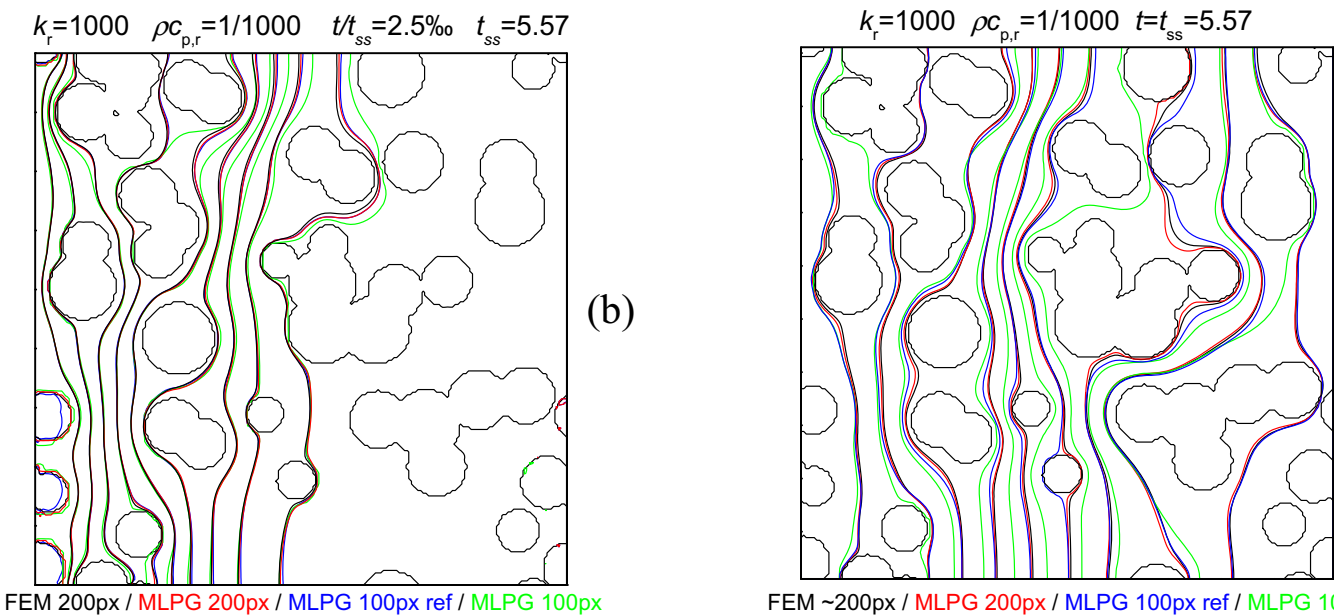

(c)

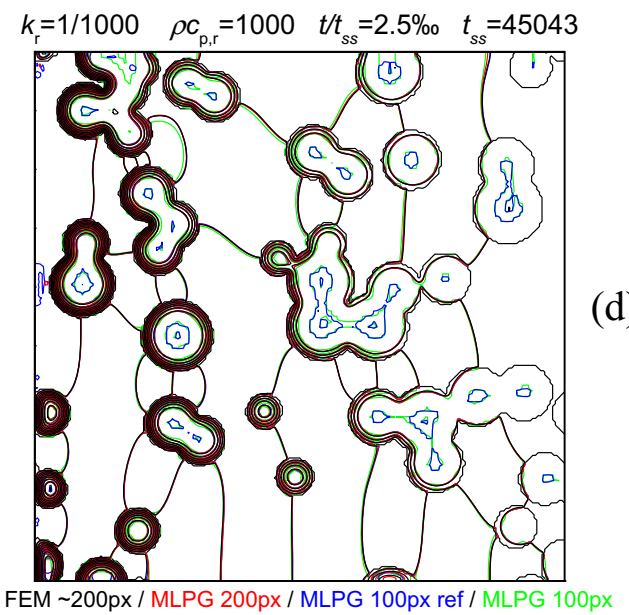

FEM 200px / MLPG 200px / MLPG 100px ref / MLPG 100px

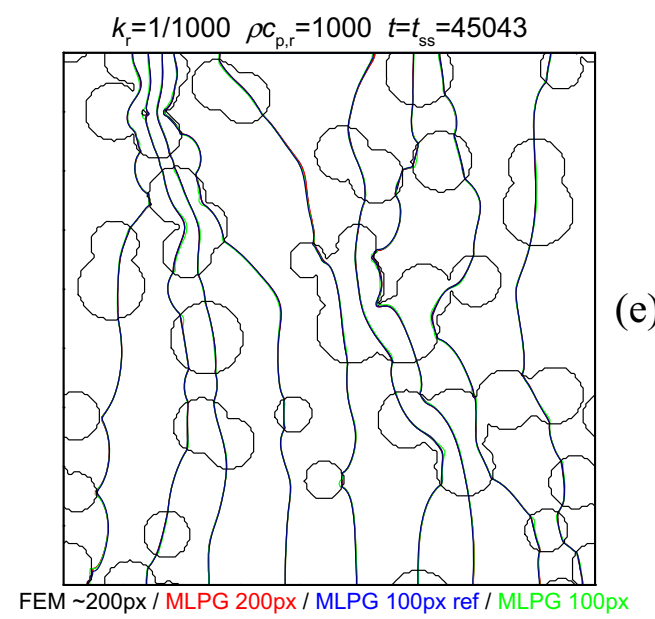

Fig. 2. (a) 1 st realization of a square enclosure with varying conductivity. White regions: medium with conductivity $k_{1}$ (exterior). Shaded regions: medium with conductivity $k_{2}$ (interior). Contours of the temperature profiles using MLPG and FE methods on a square enclosure with varying conductivity (b, d) at early time steps, and (c, e) at steady state. Conductivity of exterior medium is 1000 times (b, c) smaller or (d, e) larger than that of interior medium. Black lines: FEM solution with $\sim 200 \times 200$ resolution. Red lines: MLPG, $200 \times 200$. Green lines: MLPG, $100 \times 100$. Blue lines: MLPG, $100 \times 100$ with local refinement. (For interpretation of the references to color in this figure legend, the reader is referred to the web version of this article.)

parameters, like the $k_{r}$ and $b_{r}$ coefficients. Compared to the FEM $\sim 200$ px method, the construction phase of the MLPG approach is comparatively faster (about $1 / 3$ for 1 st order MLPG basis function) for 100 px resolution, but slower (about $3 / 2$ for 1 st order basis function, and inasmuch as 4 times for 2 nd order) for $200 \mathrm{px}$ resolution. However it is comparable and somewhat faster during implementation of refinement at $100 \mathrm{px}$. 


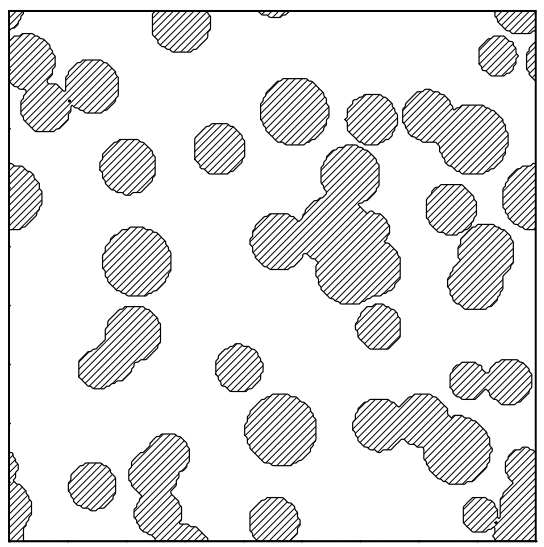

(a)

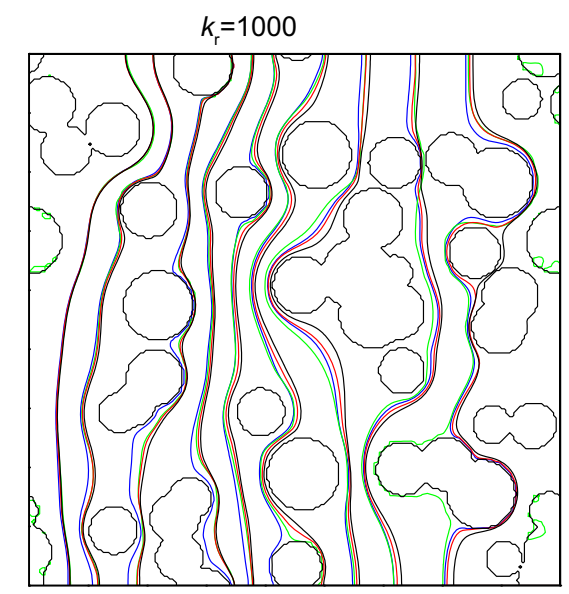

FEM 200px | MLPG-200px | MLPG-100px,ref | MLPG-100px

(b)

Fig. 3. (a) 2nd realization of a square enclosure with varying conductivity. White regions: medium with conductivity $k_{1}$ (exterior). Shaded regions: medium with conductivity $k_{2}$ (interior). (b, c) Contours of the temperature profiles using MLPG and FE methods on a square enclosure with varying conductivity at steady state Black lines: FEM solution with $\sim 200 \times 200$ resolution. Red lines: MLPG, $200 \times 200$. Green lines: MLPG, $100 \times 100$. Blue lines: MLPG, $100 \times 100$ with local refinement. (For interpretation of the references to color in this figure legend, the reader is referred to the web version of this article.)

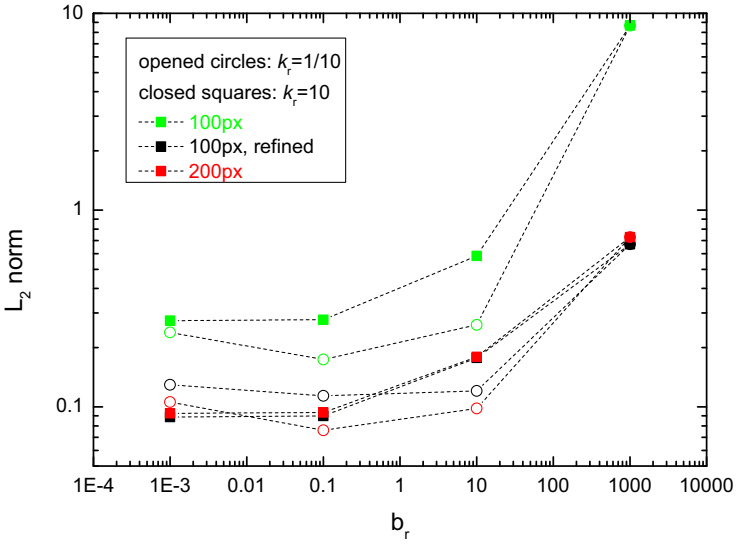

(a)

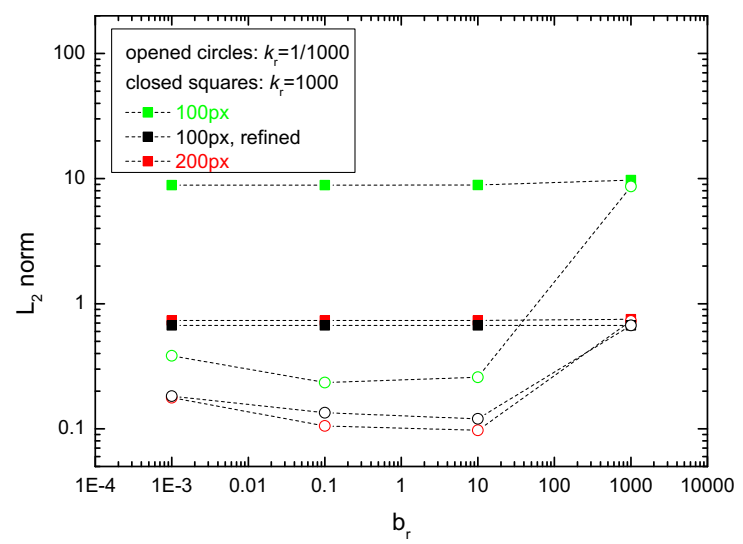

(b)

Fig. 4. Error in the $L_{2}$ norm values calculated with standard and with locally refined MLPG methods (at steady state), for (a) small and (b) large values of the temperature-independent conductivity coefficient ratio, $k_{\mathrm{r}}$, as functions of the temperature-dependent conductivity coefficient ratio, $b_{\mathrm{r}}$, as defined in Eq. (13), using rectangular integration sites. 


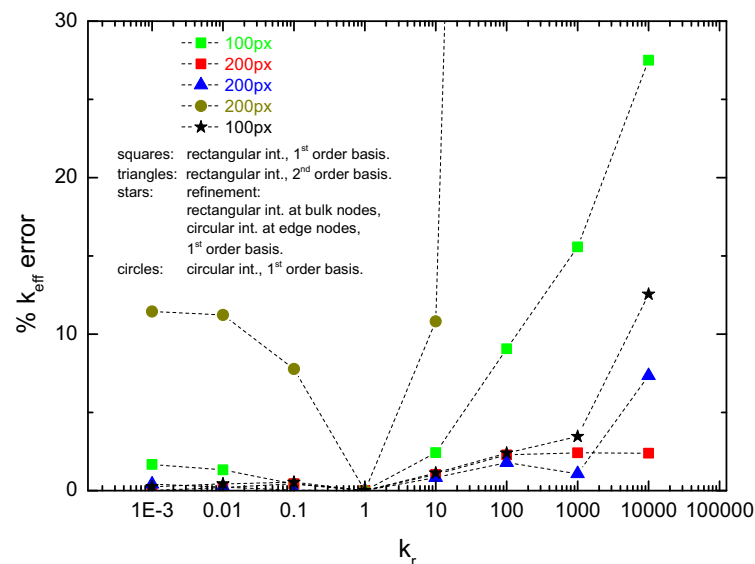

(a)

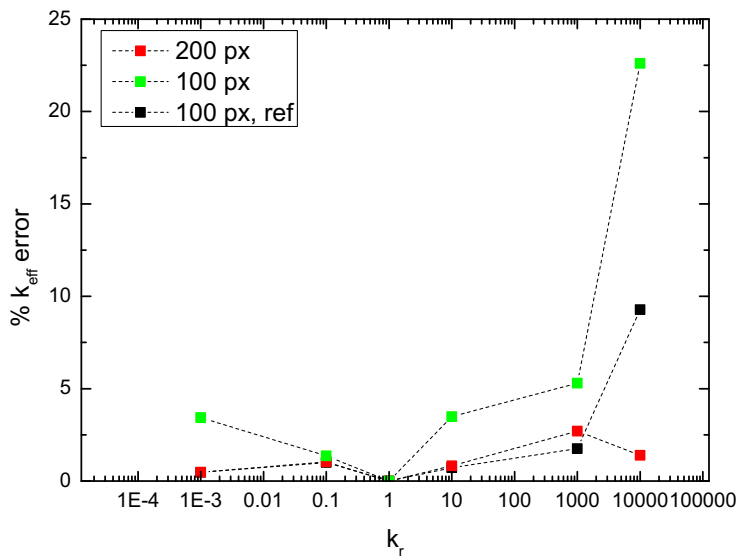

(c)

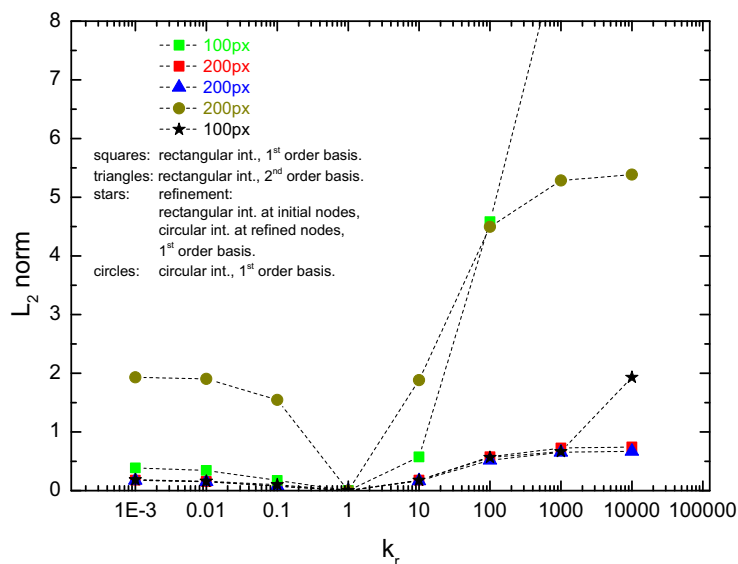

(b)

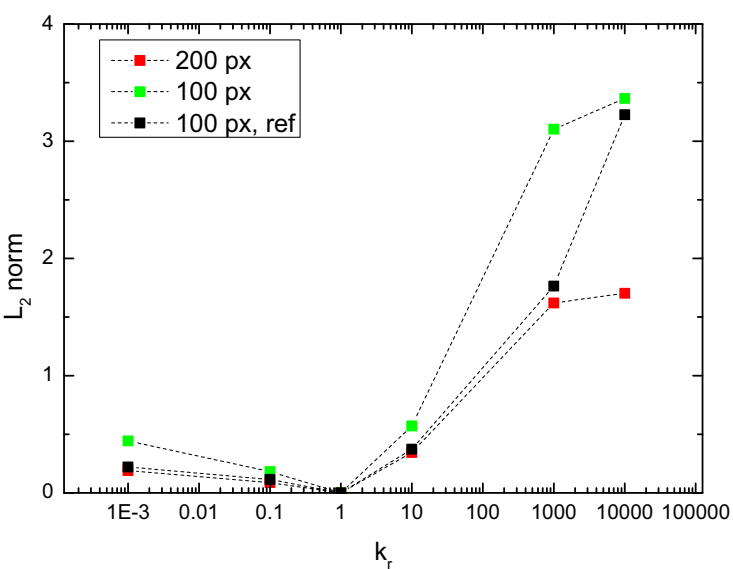

(d)

Fig. 5. Error in (a, c) the $k_{\text {eff }}$ values and (b, d) the $\mathrm{L}_{2}$ norms calculated with the MLPG methods at steady state, with and without refinement, compared with the $k_{\text {eff }}$ values of a conventional FE method, for (a, c) the first and (b, d) the second realization of the sample, and (a-b) for 1st and 2nd order MLPG methods, using rectangular and circular integration sites.

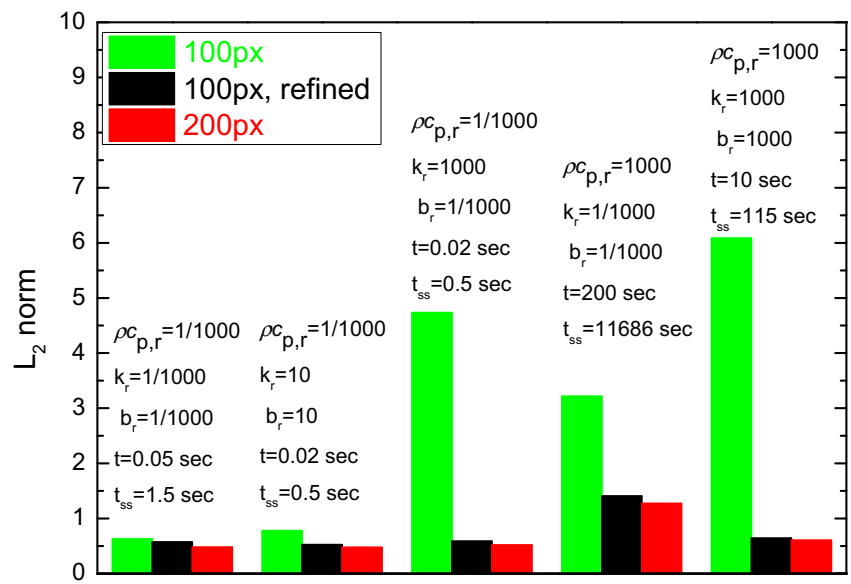

Fig. 6. Effect of refinement on the error of the L2 norm, for a range of parameters examined at early stages of transient heat conduction simulations; the temperature-independent conductivity coefficient ratio $\left(k_{\mathrm{r}}\right)$, the volumetric heat capacity range $\left(\rho c_{\mathrm{p}, \mathrm{r}}\right)$, and the temperature dependence of conductivity $\left(b_{\mathrm{r}}\right)$. 

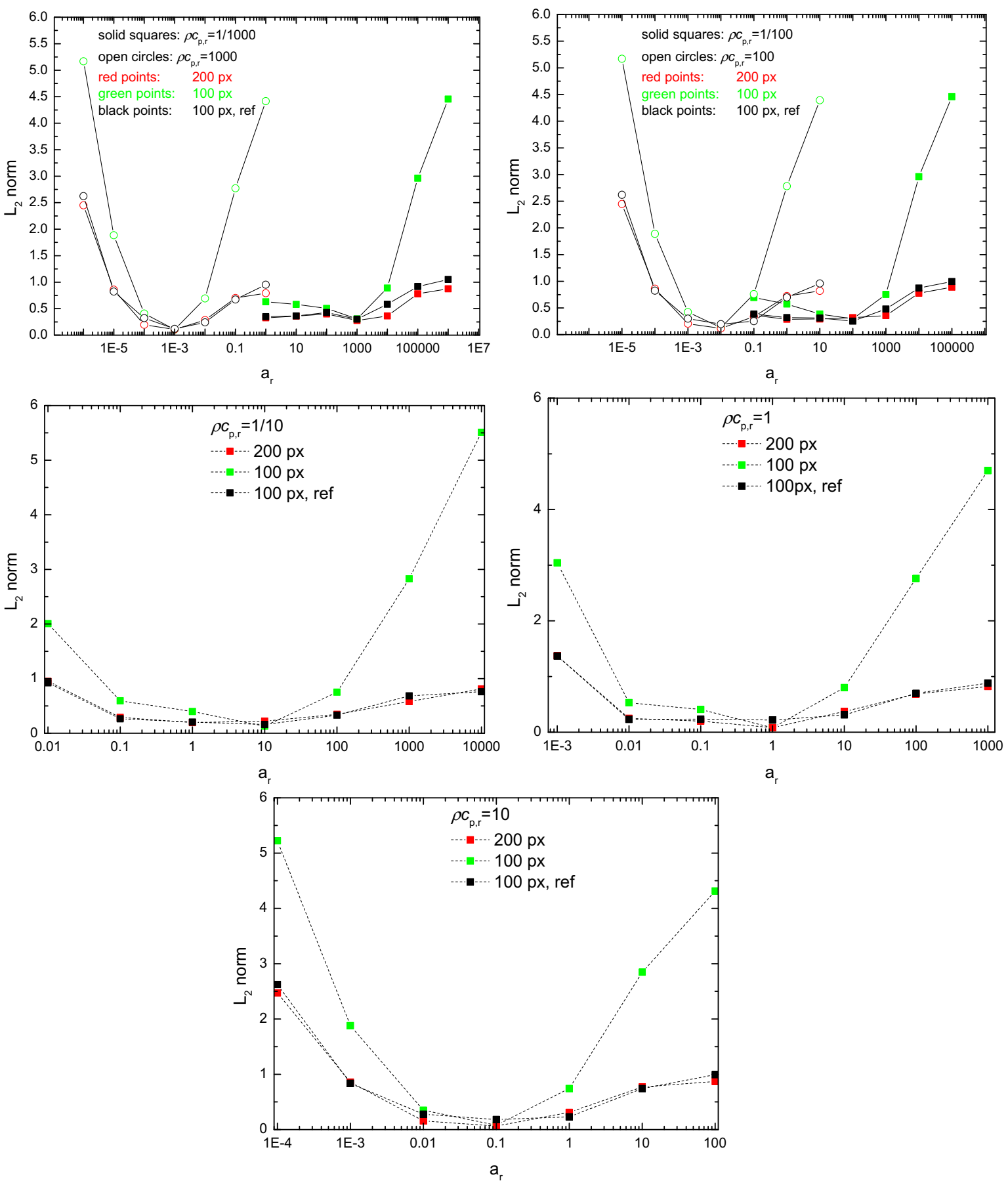

Fig. 7. Error in $L_{2}$ norm as a function of the thermal diffusivity at increasing volumetric heat capacity ratios.

Average time estimations of solution stage are shown in Table 2, for steady-state and early stages of transient MLPG and FE methods used in the present work, for both constant and temperature-varying conductivities. Times are averaged for $k_{r}$ over the (1/1000 to 10000) range, and $b_{r}$ in the (1/1000 to 10000) range, with maximum 4 iterations necessary for convergence. During solution, the advantage of MLPG approximation is evident for both 1st and 2nd order basis functions and for both 200 px and 100 px refined resolution, compared to the FEM performance of 200 px. The MLPG refinement developed and implemented here adds little to the overall computational time, own basically to the local characteristics of the refinement procedure, and surpasses the FE method in computational efficiency. 


\section{Table 1}

Average time estimations of construction stages, i.e. calculation of shape functions and integrals, for steady-state and transient MLPG methods, and for the mesh generation of the FE method used in the present work.

\begin{tabular}{|c|c|c|}
\hline Method & $\begin{array}{l}\text { Construction time steady state } \\
\text { (line integrals) (s) }\end{array}$ & $\begin{array}{l}\text { Construction time dependence } \\
\text { (line + surface integrals) (s) }\end{array}$ \\
\hline MLPG 1st order, 100 px per dim. (10,101 nodes) & 13 & $13+32$ \\
\hline $\begin{array}{l}\text { MLPG refined locally at double resolution, } 1 \text { st order, initially } 100 \\
\text { px per dim. }(23,727 \text { nodes })\end{array}$ & 28 & $28+59$ \\
\hline MLPG 1 st order, 200 px per dim. (40,401 nodes) & 48 & $48+93$ \\
\hline MLPG 2nd order, 200 px per dim. (40,401 nodes), 40401 nodes & 130 & $130+305$ \\
\hline FEM, $\sim 200$ px per dim. (41,769 elements) & 33 & $33+0$ \\
\hline
\end{tabular}

Table 2

Average time estimations of solution stage, for steady-state (upper part) and early stages of transient (lower part) MLPG and FE methods used in the present work, for both constant (middle column) and temperature-varying conductivity (right column).

\begin{tabular}{|c|c|c|}
\hline Method & Solution time steady state (s) & Solution time steady state $k=k(T)(\mathrm{s})$ \\
\hline MLPG 1 st order, 100 px per dim. (10,101 nodes) & 0.12 & 0.59 \\
\hline $\begin{array}{l}\text { MLPG refined locally at double resolution, } 1 \text { st order, initially } \\
100 \text { px per dim. }(23,727 \text { nodes })\end{array}$ & 0.3 & 1.8 \\
\hline MLPG 1 st order, 200 px per dim. (40,401 nodes) & 0.56 & 3.4 \\
\hline \multirow[t]{2}{*}{ FEM, $\sim 200$ px per dim. (41,769 elements) } & 6 & 13 \\
\hline & $\begin{array}{l}\text { Solution time time dependence } \\
(1 / 25 \text { of steady state) }(s)\end{array}$ & $\begin{array}{l}\text { Solution time time dependence } k=k(T) \\
(1 / 25 \text { of steady state) (s) }\end{array}$ \\
\hline MLPG 1st order, 100 px per dim. (10101 nodes) & 7 (72 iterations) & 15 (55 iterations) \\
\hline $\begin{array}{l}\text { MLPG refined locally at double resolution, } 1 \text { st order, initially } \\
100 \text { px per dim. }(23,727 \text { nodes })\end{array}$ & 20 (73 iterations) & 48 (56 iterations) \\
\hline MLPG 1 st order, 200 px per dim. (40,401 nodes) & 33 (73 iterations) & 75 (55 iterations) \\
\hline FEM, $\sim 200$ px per dim. (41,769 elements) & 81 (80 iterations) & 486 (58 iterations) \\
\hline
\end{tabular}

\section{Conclusions}

In the present work, the ability of Meshless methods to apply local refinement with minimum additional burden and manual effort, which is one of their key advantages in solving heat conduction problems, was shown to offer better description of the effective conductance and temperature. Local refinement surpassed unrefined simulations nearly an order of magnitude in accuracy, in the range of parameters examined here, while it optimized the solution in computational cost. Application of 2nd order interpolation scheme was shown to increase the computational time; however, it had little effect on the errors of transient problems.

In addition, novel specific rectangular-type integration sites have been introduced during MLPG integration. Typical MLPG and other Meshless formulations usually apply circular-type integration sites during domain integration, which either do not fully cover the area of the problem domain or their application produce systematic overlapping, and this have considerable effect the stability of the method. In contrast, the rectangular ones introduced here led to zero overlapping and had notable improvement on the stability of the method, particularly at elevated conductivity ratios.

Previous applications of the MLPG method to heat conduction studies [12-15] have targeted linearly-variable problems with the application of the Laplace transform for the elimination of the time dependence of the differential equation using ordinary circular-type integration sites around uniformly distributed nodes. The present MLPG approach successfully addressed non-linear problems of elevated complexity, i.e. at variable conductivity ratios covering four orders of magnitude, using local refinement, and achieved increased robustness with direct time integration using specific semi-explicit timestepping formulation and sophisticated MLPG integration sites that cover the whole domain.

Ordinary FE methods have been utilized for validation and benchmarking purposes of these non-linear problems. Comparing the computational efficiency between the MLPG and the FE methods, the MLPG methods suffered from increased construction times, when weighed against similar FE methods. However, during solution the advantage of MLPG approximation was evident for any combination of basis order and resolution, compared to the efficiency of FEM approaches. Due to its local character, the MLPG refinement developed and implemented here using a combination of rectangular and circular integration sites adds little to the overall computational time, and exceeds in computational competence both unrefined MLPG and FE methods of globally similar resolution, and even refined FEM ones, i.e. with the application of boundary layers around conductivity boundaries.

\section{Acknowledgments}

This research has been co-financed by the European Union (European Social Fund - ESF) and Greek national funds through the Operational Program "Education and Lifelong Learning" of the National Strategic Reference Framework (NSRF) - Research Funding Program: ARCHIMEDES III. Investing in knowledge society through the European Social Fund. 


\section{References}

[1] A. Bejan, Heat Transfer, John Wiley \& Sons, NY, 1993.

[2] S. Mahjoob, K. Vafai, Analytical characterization of heat transport through biological media incorporating hyperthermia treatment, Int. J. Heat Mass Transfer 52 (2009) 1608-1618.

[3] T.C. Chiam, Heat transfer with variable conductivity in a stagnation-point flow towards a stretching sheet, Int. Commun. Heat Mass Transfer 23 (1996) 239-248.

[4] M. Arunachalam, N.R. Rajappa, Thermal-boundary layer in liquid-metals with variable thermal-conductivity, Appl. Sci. Res. 34 (1978) $179-187$.

[5] G.E. Wnek, J.C.W. Chien, F.E. Karasz, M.A. Druy, Y.W. Park, A.G. MacDiarmid, A.J. Heeger, Variable-density conducting polymers: conductivity and thermopower studies of a new form of polyacetylene: (CH)x, J. Polym. Sci. Polym. Lett. Edition 17 (1979) $779-786$.

[6] B. Nayroles, G. Touzot, P. Villon, Generalizing the finite element method: diffuse approximation and diffuse elements, Comput. Mech. 10 (1992) 307318.

[7] T. Belytschko, Y.Y. Lu, L. Gu, Element-free Galerkin methods, Int. J. Numer. Methods Eng. 37 (1994) $229-256$.

[8] D. Organ, M. Fleming, T. Terry, T. Belytschko, Continuous meshless approximations for nonconvex bodies by diffraction and transparency, Comput. Mech. 18 (1996) 225-235.

[9] T. Zhu, S.N. Atluri, A modified collocation method and a penalty formulation for enforcing the essential boundary conditions in the element free Galerkin method, Comput. Mech. 21 (1998) 211-222.

[10] W.K. Liu, Y. Chen, C.T. Chang, T. Belytschko, Advances in multiple scale kernel particle methods, Comput. Mech. 18 (1996) $73-111$.

[11] G.C. Bourantas, E.D. Skouras, V.C. Loukopoulos, G.C. Nikiforidis, An accurate, stable and efficient domain-type meshless method for the solution of MHD flow problems, J. Comput. Phys. 228 (2009) 8135-8160.

[12] J. Sladek, V. Sladek, C. Zhang, Transient heat conduction analysis in functionally graded materials by the meshless local boundary integral equation method, Comput. Mater. Sci. 28 (2003) 494-504.

[13] V. Sladek, J. Sladek, M. Tanaka, C. Zhang, Transient heat conduction in anisotropic and functionally graded media by local integral equations, Eng. Anal. Bound. Elem. 29 (2005) 1047-1065.

[14] J. Sladek, V. Sladek, C. Hellmich, J. Eberhardsteiner, Heat conduction analysis of 3-D axisymmetric and anisotropic FGM bodies by meshless local Petrov-Galerkin method, Comput. Mech. 39 (2007) 323-333.

[15] J. Sladek, V. Sladek, C.L. Tan, S.N. Atluri, Analysis of transient heat conduction in 3D anisotropic functionally graded solids, by the MLPG method, CMES - Comput. Model. Eng. Sci. 32 (2008) 161-174.

[16] S.N. Atluri, T. Zhu, A new meshless local Petrov-Galerkin (MLPG) approach in computational mechanics, Comput. Mech. 22 (1998) 117-127.

[17] G.C. Bourantas, E.D. Skouras, G.C. Nikiforidis, Adaptive support domain implementation on the moving least squares approximation for mfree methods applied on elliptic and parabolic pde problems using strong-form description, CMES - Comput. Model. Eng. Sci. 43 (2009) 1-25.

[18] A.N. Kalarakis, G.C. Bourantas, E.D. Skouras, V.C. Loukopoulos, V.N. Burganos, Lattice-Boltzmann and meshless point collocation solvers for fluid flow and conjugate heat transfer, Int. J. Numer. Methods Fluids 70 (2012) 1428-1442.

[19] G.C. Bourantas, A.J. Petsi, E.D. Skouras, V.N. Burganos, Meshless point collocation for the numerical solution of Navier-Stokes flow equations inside an evaporating sessile droplet, Eng. Anal. Bound. Elem. 36 (2012) 240-247.

[20] G.C. Bourantas, E.D. Skouras, V.C. Loukopoulos, V.N. Burganos, Heat transfer and natural convection of nanofluids in porous media, Eur. J. Mech. B Fluids 43 (2014) 45-56.

[21] E.D. Skouras, G.C. Bourantas, V.C. Loukopoulos, G.C. Nikiforidis, Truly meshless localized type techniques for the steady-state heat conduction problems for isotropic and functionally graded materials, Eng. Anal. Bound. Elem. 35 (2011) 452-464.

[22] N. Karagiannakis, G.C. Bourantas, A.N. Kalarakis, E.D. Skouras, V.N. Burganos, Efficiency of the meshless local Petrov-Galerkin method with moving least squares approximation for thermal conduction applications, in: T. Simos (Ed.) ICNAAM 2013, Rhodos, Greece, 2013.

[23] S.N. Atluri, Z.D. Han, A.M. Rajendran, A new implementation of the meshless finite volume method, through the MLPG "Mixed" approach, CMES Comput. Model. Eng. Sci. 6 (2004) 491-513.

[24] X.H. Wu, W.Q. Tao, Meshless method based on the local weak-forms for steady-state heat conduction problems, Int. J. Heat Mass Transf. 51 (2008) 3103-3112. 